\title{
Novel method for thermal conductivity measurement through flux signal deconvolution
}

\author{
Heinrich Badenhorst ${ }^{*}$, Carl Sandrock \\ SARChI Chair in Carbon Materials and Technology, Department of Chemical Engineering, University \\ of Pretoria, Lynnwood Road, Pretoria, 0002, South Africa
}

\section{Highlights}

- Transient heat flux technique for thermal properties of high conductivity solids.

- Combining the use of two heat flux measurements with short heat impulse.

- The fundamental model which describes the system is used as basis.

- Optimization is used to fit the measured system impulse response.

- Validated using two commercial metals, suitable for practical, composite systems.

\begin{abstract}
Rapid thermal conductivity measurement of porous solids and composites remains a challenge. A modified steady state technique has been proposed which uses two heat flux sensors instead of one. The parameter estimation is achieved through the deconvolution of these signals and the identification of the system impulse response. A detailed derivation of the theoretically expected behaviour has been done, which provides a basis for fitting the measured impulse response. A six term expansion is required for the theoretical model to achieve full convergence. The unit requires a calibration step to measure the convective boundary condition. A signal validity check has been built into the approach through the use of the energy balance which detects any drift due to ambient losses or other factors. Through suitable choice of the mathematical algorithm rapid convergence of the non-linear fitting procedure is achieved. The parameter estimates of the standard test samples are excellent, with average errors of $2.3 \%$ for brass and $6.3 \%$ for aluminium. The system has several advantages in addition to the short measurement time, including low cost and no guard furnace or insulation requirement for room temperature measurements. The
\end{abstract}

\footnotetext{
* Corresponding author. Tel: +27 12420 4989. Fax: +27 12420 2516. E-mail: carbon@up.ac.za
} 
approach is suitable for measuring the overall behaviour of practical, composite systems.

Keywords: Energy Materials; Thermal Properties; Analytical Modelling

\section{Introduction}

High thermal conductivity materials are used in countless applications from thermal management to energy storage. Accurate knowledge of the thermal transport properties is critical for design activities as well as the rapid development of new materials. Recently novel, highly conductive, graphitic foams have been developed for these applications $[1,2]$. These materials have extensive macro and micro porosity and correspondingly low densities $\left(\sim 0.3 \mathrm{~g} . \mathrm{cm}^{-3}\right)$. The open pore structure makes them ideal for passive heat dissipation applications due to the ease of convective heat transfer. Alternatively these foams may be employed for property enhancement of substances having high energy capacity but low thermal conductivity. Such composites are ideal for thermal energy storage applications.

Many techniques have been developed over the years to measure the thermal properties of different materials, each with its advantages and disadvantages. These may be broadly classified into two main categories: transient and steady state. Due to their short measurement time transient methods such as the line source [3-5], hot strip [6-8], plane source [9-11] and laser flash [12,13] methods have been widely used. A closely related method to the hot-wire and hot-strip techniques is the $3 \omega$ method [14] which uses a frequency based analysis to measure thermal conductivity rather than a temporal approach.

However, these methods do suffer from practical issues when measuring the properties of porous solids. This is especially true for composite or multi-layer materials which are more representative of practical arrangements [15]. These may include contact issues, due to the rough surface of porous solids the number of contact points are reduced leading to inaccurate results unless large sample sizes are used. Sample penetration may also be limited $[16,17]$ which is a problem for composites which are inhomogeneous and often have different surface properties due to 
anisotropic processing. The laser flash method for example is only applicable to suitably thin discs or films as stipulated by the ISO standard 22007-4:2008. For some composites such as foams which are very brittle, it is difficult to obtain very thin slices.

In addition, the transient hot strip method for example also suffers from the use of an approximate relationship for the property estimation rather than a rigorous theoretical solution [18]. Steady state methods on the other hand have a sound theoretical basis and many have been developed according to the American Society for Testing and Materials (ASTM) standards, for example C177 (Standard Test Method for Steady-State Heat Flux Measurements) and C518 (Standard Test Method for Steady-State Thermal Transmission Properties). One of these, ASTM E1530 (Guarded Heat Flow Meter Method), involves the use of plates with fixed temperatures in conjunction with a heat flux sensor. The primary problem with a steady state method is the time required to reach steady state [19]. For the aforementioned method a waiting time of at least a few hours is recommended and as such a guard furnace is required to minimize any heat loss during the measurement. In addition to this, steady state methods are generally not advocated for materials that have a high thermal conductivity. This is because of the small temperature gradients developed and the ASTM E1530 is not recommended for materials having a thermal resistance lower than $1 \times 10^{-3} \mathrm{~m}^{2} \cdot \mathrm{K} \cdot \mathrm{W}^{-1}$.

To overcome these limitations, a novel modification of the ASTM 1530 technique is proposed in this paper which involves the use of two heat flux sensors rather than one. This method retains the robustness of the steady state technique, making it possible to measure highly conductive, porous solids and composites, whilst significantly reducing the measurement time. What makes the approach unique is the use of flux signal deconvolution to find the impulse response of the system, which is in turn used to determine the thermal conductivity. In process identification terminology a transient system model may be represented as shown in Figure 1. 


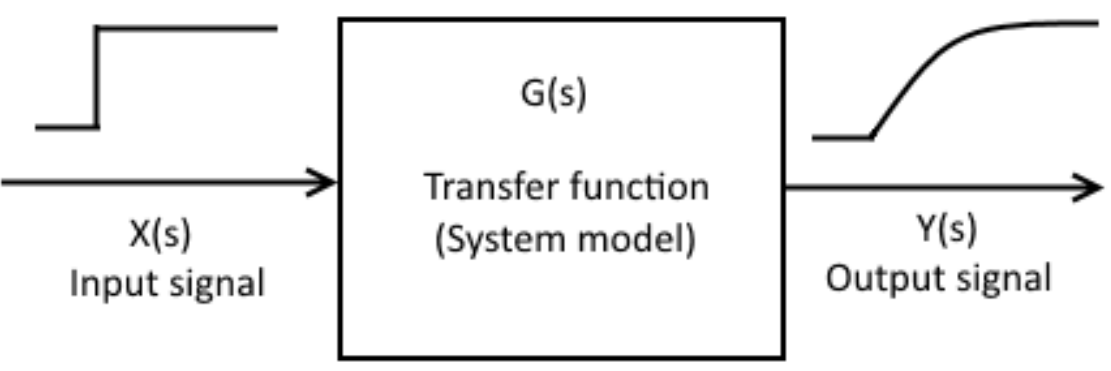

Figure 1: System model

In this case the input signal is a step and the output signal is a simple first order response. However, the input signal is arbitrary and can be an impulse function in which case the output signal is termed the impulse response of the system. If the system model is unknown it can be obtained from the input and output signals. However, this is not a straightforward division of the time based signals. In real measurement systems the input signal is not a continuous measurement as shown in Figure 1 but is instead comprised of discrete values sampled at a given frequency. For this reason the input signal may be viewed as a set of impulses and the impulse response of the system may be obtained by the frequency domain decomposition and deconvolution of input and output signals [20]. This is true irrespective of the overall observed shape of the input signal which still remains arbitrary. In the case of this investigation the input and output signals of the system are the flux measurements.

To avoid the use of an approximate relationship a fundamental model was first developed for the proposed measurement technique. This enables a detailed analysis of the expected impulse response of the system. Unfortunately, this function is very non-linear and cannot be cast into a form which would explicitly give the desired parameter estimate. For this reason it is necessary to employ an optimization approach to find the appropriate parametric value which fits the impulse response of the experimental data. The objective of this investigation is to develop a suitable methodology for parameter estimation using the new technique. This includes signal validation, model formulation and the choice of optimization algorithm. The approach is validated using two, high thermal conductivity, metallic samples with known thermal properties. This work represents the first step in the development of a technique for the overall heat transfer coefficient measurement of practical, composite systems. 


\section{Experimental}

In essence the sample is sandwiched between two heat flux sensors with a hot source and cold sink above and below respectively. The experimental setup is shown schematically in complete detail, in Figure 2 below.

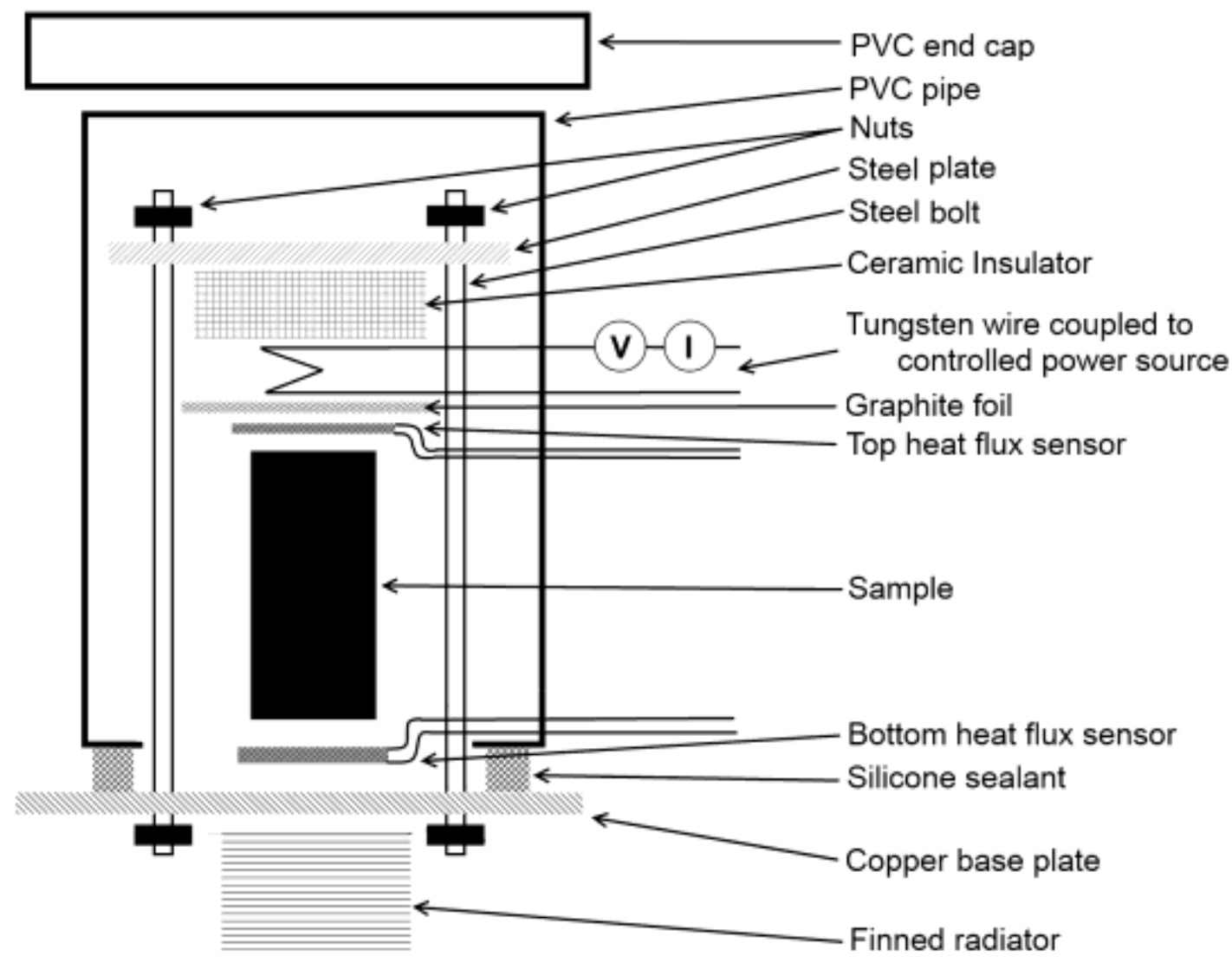

Figure 2: Experimental setup for transient heat flux measurement

The assembly is clamped using steel bolts between a steel top and copper base plate to ensure good contact. The entire system is placed in a circulating water bath at a constant temperature which acts as the cold sink. The PVC pipe open spaces are filled with glass fibre wool for additional insulation. The heating element is linked to a controlled power source (maximum power $1 \mathrm{~kW}$ ) and is shielded from the steel plate using a ceramic insulator. The element is a tungsten wire $(\mathrm{L}=10 \mathrm{~cm}, \mathrm{D}=100$ $\mu \mathrm{m})$ placed in a zigzag arrangement on top of a very thin, highly conductive graphite foil. The graphite foil has comparatively low thermal conductivity through the plane but very high in plane conductivity $\left(\sim 1500 \mathrm{~W} \cdot \mathrm{m}^{-1} \cdot \mathrm{K}^{-1}\right)$. This ensures a very low thermal gradient with a virtually homogenous temperature distribution and hence flux across the sample top surface. The graphite foil (SS1500 eGRAF® Spreadershield ${ }^{\mathrm{TM}}$ ) 
was obtained from a commercial supplier, GrafTech International (U.S.A.). Since the input flux signal is measured, there is no requirement for an exact, controlled shape. For a single experimental run the power source is set to maximum output and is turned on only for a few seconds before being switched off.

The heat flux sensors (HFS-4) were obtained from OMEGA Engineering, Inc (U.S.A.). The sensor is a thin film comprised of a $50+$ junction thermopile bonded to either side of a Kapton barrier, which has known thermal characteristics. Since the heat transfer rate is directly proportional to the temperature difference across the thermal barrier, the exact rate of transfer can be calculated by measuring this difference. The sensor is $35 \mathrm{~mm}$ by $28.5 \mathrm{~mm}$ with a reported sensitivity of 2 $\mu \mathrm{V} .\left(\mathrm{W} . \mathrm{m}^{-2}\right)^{-1}$ and has an extremely low thermal resistance. All interfaces are coated with a thin film of silicon heat transfer compound (Unick Chemical Corp, Taiwan) to ensure surface homogeneity. For testing, brass (CDA 385) and aluminium (6082) rods $($ diameter $=20 \mathrm{~mm})$ of different lengths $(25,50,80,100 \mathrm{~mm})$ were obtained from Non-Ferrous Metal Works, Pty (South Africa). The composition and relevant physical properties for these materials given by the supplier are listed in Table 1 and 2 respectively.

Table 1: Metal compositions (weight \%)

\begin{tabular}{|c|c|c|}
\hline Element & Brass & Aluminium \\
\hline $\mathrm{Cu}$ & $55-59$ (nominal 57) & 0.1 \\
\hline $\mathrm{Fe}$ & 0.35 & 0.5 \\
\hline $\mathrm{Pb}$ & $2.5-3.5($ nominal 3) & 0.2 \\
\hline $\mathrm{Zn}$ & Remainder (nominal 40) & $0.6-1.2$ \\
\hline $\mathrm{Mg}$ & & $0.7-1.3$ \\
\hline $\mathrm{Si}$ & & $0.4-1.0$ \\
\hline $\mathrm{Mn}$ & & 0.1 \\
\hline $\mathrm{Ti}$ & & 0.25 \\
\hline $\mathrm{Cr}$ & & max 0.5 \\
\hline $\mathrm{Other}$ & & Remainder \\
\hline $\mathrm{Al}$ & & \\
\hline
\end{tabular}


Table 2: Metal physical properties

\begin{tabular}{|c|c|c|c|}
\hline & $\begin{array}{c}\text { Density } \\
\left(\mathrm{kg} \cdot \mathrm{m}^{-3}\right)\end{array}$ & $\begin{array}{c}\text { Heat Capacity } \\
\left(\mathrm{J} \cdot \mathrm{kg}^{-1} \cdot \mathrm{K}^{-1}\right)\end{array}$ & $\begin{array}{c}\text { Thermal Conductivity } \\
\left(\mathrm{W} \cdot \mathrm{m}^{-1} \cdot \mathrm{K}^{-1}\right)\end{array}$ \\
\hline Brass & 8470 & 377.1 & 122.9 \\
\hline Aluminium & 2700 & 880 & $180-189$ \\
\hline
\end{tabular}

For the aluminium sample an intermediate value for the thermal conductivity of $185\left(\mathrm{~W} \cdot \mathrm{m}^{-1} \cdot \mathrm{K}^{-1}\right)$ was chosen as the theoretical value.

\section{Theoretical model}

The ASTM method E1530 together with the proposed modification are shown in Figure $3 \mathrm{~A}$ and $\mathrm{B}$. In addition the simplified system used for modelling is illustrated in Figure 3 C.

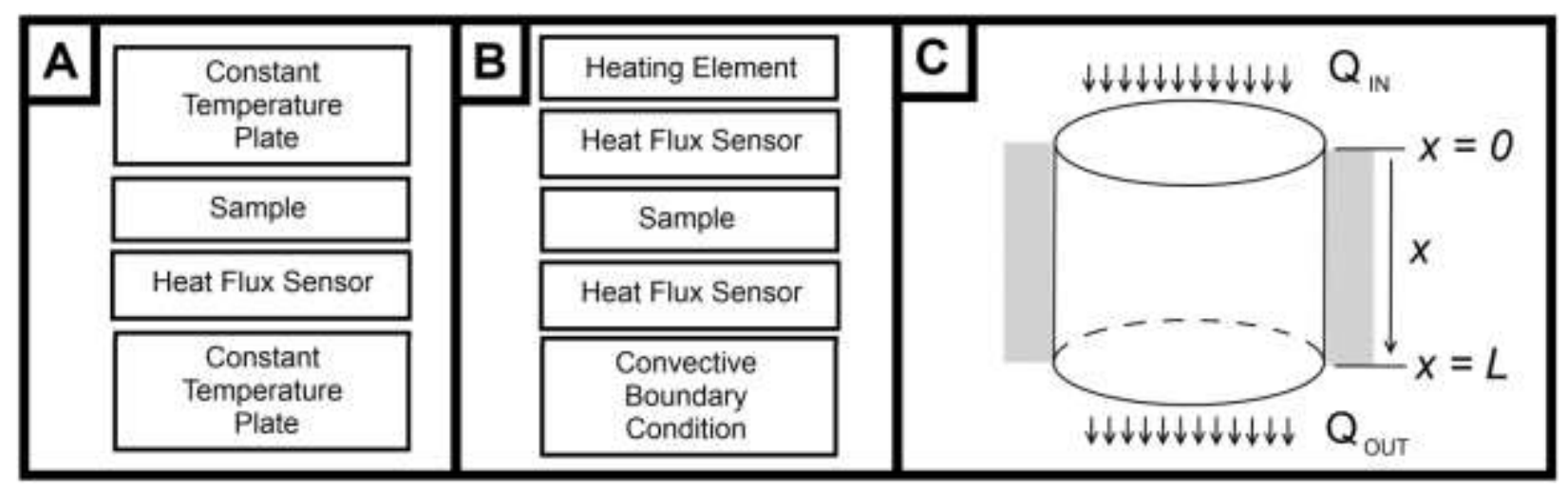

Figure 3: (A) ASTM E1530 (B) Proposed modification (C) Simplified model of the experimental setup

To model the system a solution to the general heat equation is sought:

$$
\frac{\partial u}{\partial t}=\alpha \nabla^{2} u
$$

Where $u$ is an arbitrary field variable, in this case normalized temperature and $\alpha$ is the thermal diffusivity. Since heat flow into the cylinder is assumed to be invariant, this reduces to a one dimensional problem for finding $u(x, t)$ where:

$$
u_{t}=\alpha u_{x x}
$$

In this case the subscript indicates a derivative with respect to that variable. Once the solution for $u$ is found, the value of $u_{x=L}$ can be determined, as measured by the output flux sensor. The impulse response of the system can be obtained as the time derivative of the unit step response, which is: 


$$
-u_{x}(0, t)= \begin{cases}0, & t \leq 0 \\ 1, & t>0\end{cases}
$$

With the initial condition, $u(x, 0)=0$ and boundary conditions $-u_{x}(0, t)=1$ and $u_{x}(L, t)=-\frac{h}{k} u(L, t)$, where $h$ and $k$ are the convective heat transfer coefficient and thermal conductivity respectively. As shown in detail in Appendix A, this problem can be solved to find the impulse response of the system as:

$$
u_{x t}=\sum_{n=1}^{\infty} A_{n} \alpha \lambda_{n}^{3} e^{-\alpha \lambda_{n}^{2} t} \sin \left(\lambda_{n} L\right)
$$

with $A_{n}=-\frac{4}{2 L \lambda_{n}^{2}+\lambda_{n} \sin \left(2 L \lambda_{n}\right)}$ and $\lambda_{n}$ the roots of $\lambda \sin (\lambda L)=\frac{h}{k} \cos (\lambda L)$.

This solution requires the latter expression to be solved to find a specific eigenvalue, $\lambda_{n}$. Unfortunately no closed form solution of this equation can be found, so numerical methods are required to enumerate the solutions. A number of possible forms present themselves to solve this equation. It was found that the form $\sin (\lambda \mathrm{L})-$ $\frac{\mathrm{h}}{\mathrm{k} \lambda} \cos (\lambda \mathrm{L})$ led to the best numeric behaviour, as shown in Figure 4.

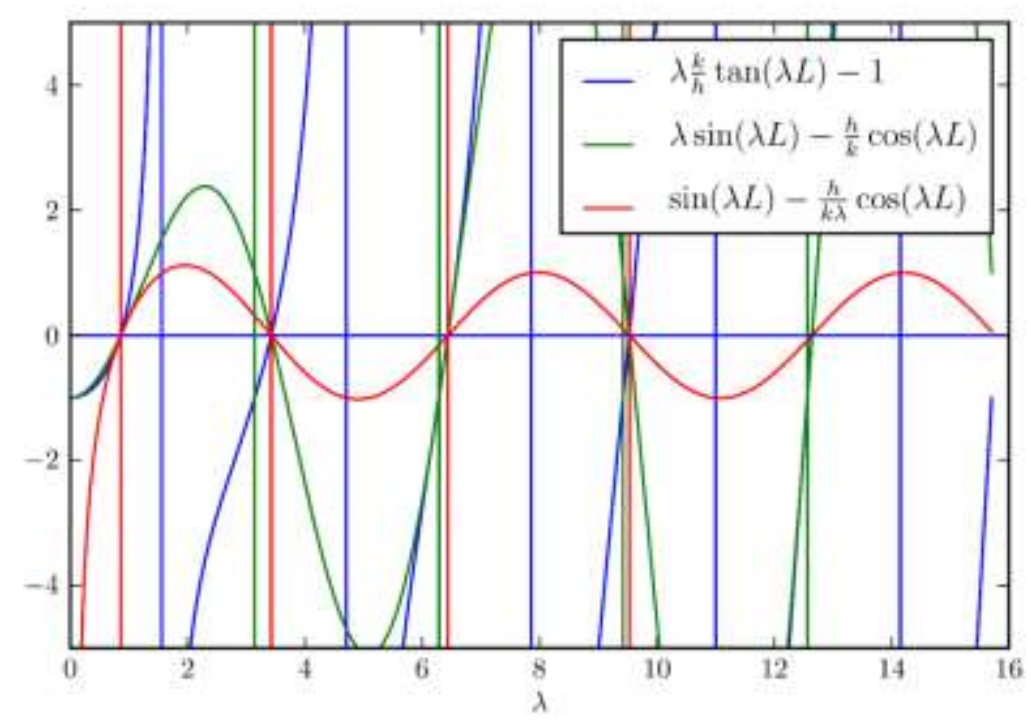

Figure 4: Different formulations of the differential function eigenvalues.

It can be seen that this form is bounded and quickly approximates $\sin (\lambda L)$ as $\lambda$ grows. This allows for efficient solution using Ridder's method [21]. For the theoretical investigation the convective heat transfer coefficient is arbitrarily set to $\left(1000 \mathrm{~W} \cdot \mathrm{m}^{-2} \cdot \mathrm{K}^{-1}\right)$. Since all other variables are known for the brass test material, the 
theoretically expected impulse responses can be calculated. This is done for a brass rod of length $80 \mathrm{~mm}$ in response to a unit impulse of 5 seconds. The result for a ten term $(n=10)$ expansion is plotted in Figure 5.

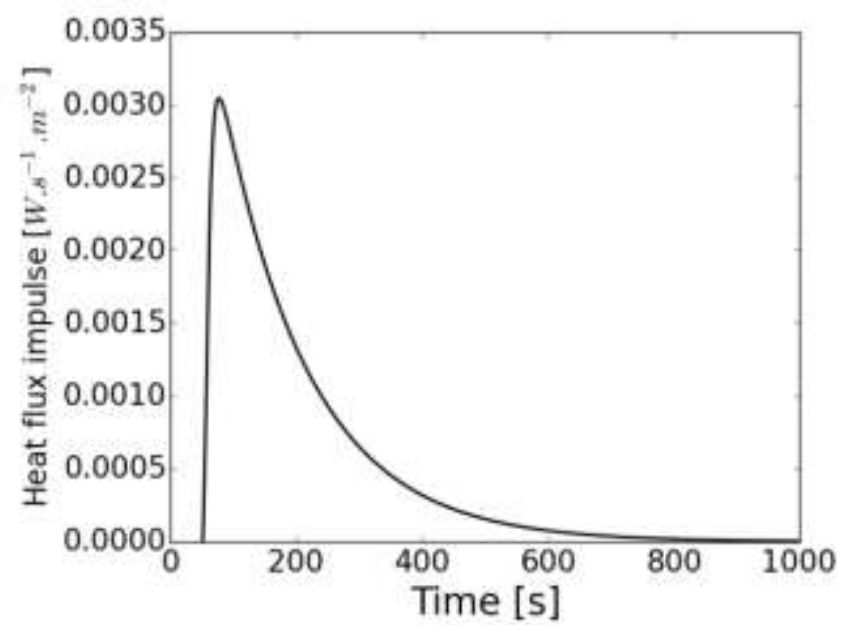

Figure 5: Theoretical impulse response for $80 \mathrm{~mm}$ brass rod $(n=10)$

The signal has been time shifted by +50 seconds for better visualization. The dynamic response of the system is as would be expected: initially the exiting heat flux rapidly increases as the influx is instantly set to a value of one. When the input is removed after only five seconds the response gradually decays as the accumulated thermal energy is dissipated. It is interesting to explore the theoretically predicted behaviour as the number of terms $(n)$ is varied, demonstrated in Figure 6.

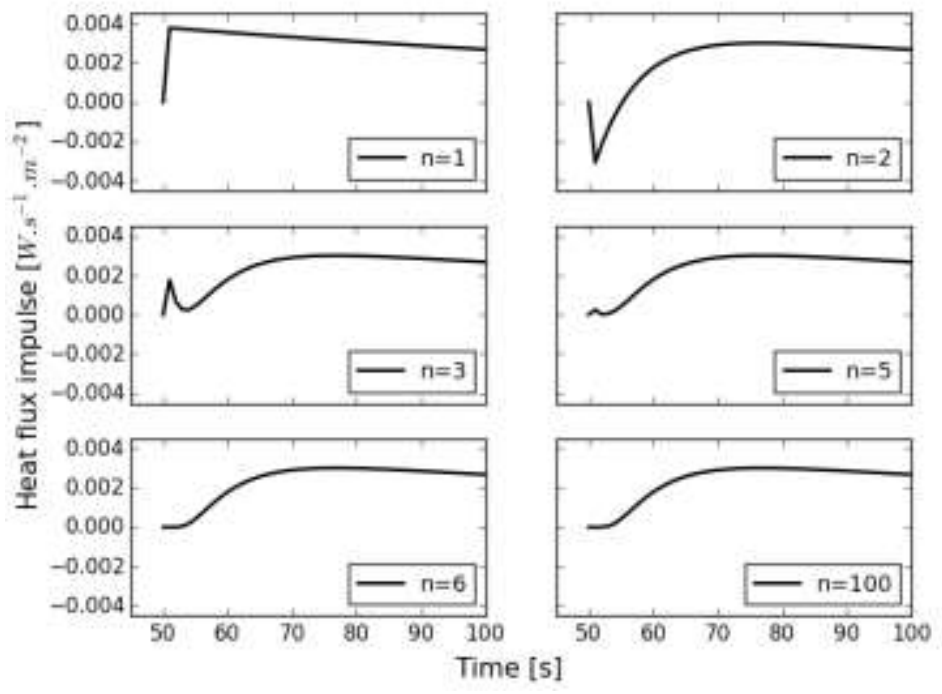

Figure 6: Variation in theoretical prediction with the number of terms 
For a single term the prediction instantly jumps to a high value after which it undergoes exponential decay. As the number of terms is increased the predictions oscillate slightly but rapidly converge to the prediction generated by a large number of terms $(n=100)$. By increasing the number of terms to five the prediction only shows a slight deviation from the large term prediction at around one second after initiation. Thus only six terms are required to achieve full conversion to the high order model. This implies that the model can be solved very rapidly to assess experimental data. Using suitable approximations it is possible to classify the behaviour exhibited by the first $(n=1)$ order prediction. If the first order Taylor approximations are used to represent the trigonometric functions it can easily be shown that equation (4) reduces to:

$$
u_{x t}=A_{1} \alpha \lambda_{1}^{3} \sin \left(\lambda_{1} L\right) e^{-\frac{h t}{\rho C_{P} L}}
$$

By noting that for a one dimensional shape the length $L$ is the characteristic length (or the volume to surface area ratio), this expression can be written in the more familiar form:

$$
u_{x t}=C e^{-B i F o}
$$

Where $B i$ and $F o$ are the Biot and Fourier numbers respectively and the constant $C$ is determined by the system parameters. Thus the single term expansion of the theoretical model represents the lumped capacitance approximation of the system.

\section{Results and discussion}

The first step in the experimental procedure is to determine the convective heat transfer coefficient $(h)$ of the setup, which is equivalent to calibrating the unit. To achieve this, a brass test sample of length $80 \mathrm{~mm}$ was tested using the device. Next the model parameters are set to the values for brass and the initial guess for the coefficient is initialised as 1 . The final value of $h$ is found by minimising the sum of the squares of the error between the model prediction and the experimentally determined impulse response, as given in Figure 7. 


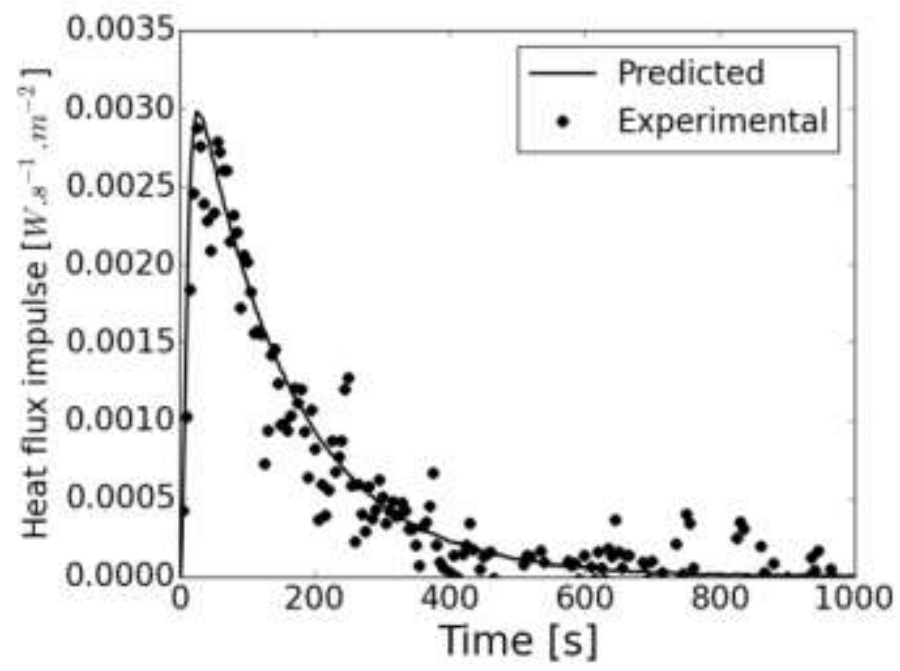

Figure 7: Calibration of experimental setup

The result shown is for a heat transfer coefficient of $h=1059 \mathrm{~W} \cdot \mathrm{m}^{-2} \cdot \mathrm{K}^{-1}$. If the system is operating consistently, this value should not change over subsequent experiments and this is indeed found to be the case. In addition, this value is in line with expectations for a liquid system under forced convection. It also implies that the system is operating as close as can be expected to an isothermal boundary condition without the need for expensive temperature controlled cooling devices. To achieve the fit a suitable algorithm must be chosen. Given the non-linearity of the problem to ensure rapid convergence the L-BFGS method as implemented in Scipy [22, 23] was used. The whole data-processing chain was implemented in Python using the SciPy routines. The residual function was well-behaved once the parameters had been scaled to be of similar magnitude.

To validate the approach the $h$ value is kept constant for all subsequent fits, whilst the length and composition of the metal rod is varied. During these fits the thermal conductivity is varied to achieve the optimal result, the thermal diffusivity is calculated using the known reference values of heat capacity and density for the material. Shown in Figure 8 are the results for brass rods of varying length. 


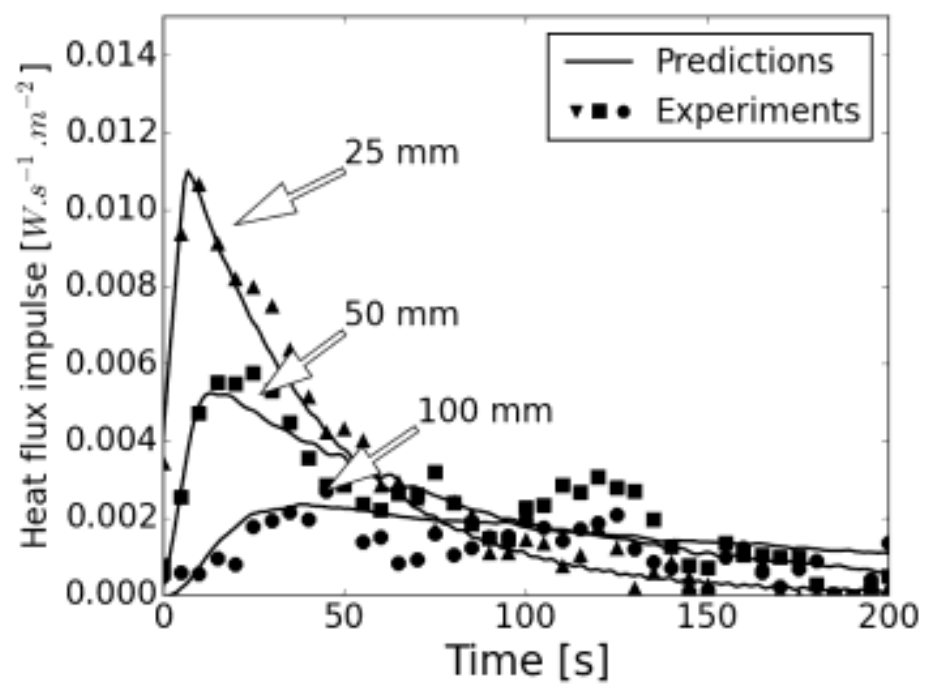

Figure 8: Brass rod test impulse response results

As can be seen from the figure the signals are very noisy due to the frequency domain division required by the deconvolution. In addition, an effort was made to keep the experimental time as short as possible. Thus the pulse time was kept to only a few seconds and consequently the signal to noise ratio is quite high. Nonetheless, the fitted values of thermal conductivity are within an average error of only $4.4 \%$ of the theoretical value for brass with a standard deviation of $2.5 \%$. The full results are given in Table 3 .

Table 3: Thermal conductivity results

\begin{tabular}{|c|c|c|c|c|}
\hline Size $(\mathrm{mm})$ & Brass & Error (\%) & Aluminium & Error (\%) \\
\hline 25 & 110.8 & 1.9 & 200.1 & 8.1 \\
\hline 50 & 105.1 & 6.9 & 171.1 & 7.5 \\
\hline 100 & 107.9 & 4.4 & 191.0 & 3.3 \\
\hline
\end{tabular}

Thus parameter estimation is very good and the results demonstrate that the convective boundary condition has remained constant throughout. The time based predictions can also be easily calculated using the expression for the flux (temperature spatial derivative) before the temporal derivative is taken, given in Appendix A. These predictions are plotted in Figure 9. 


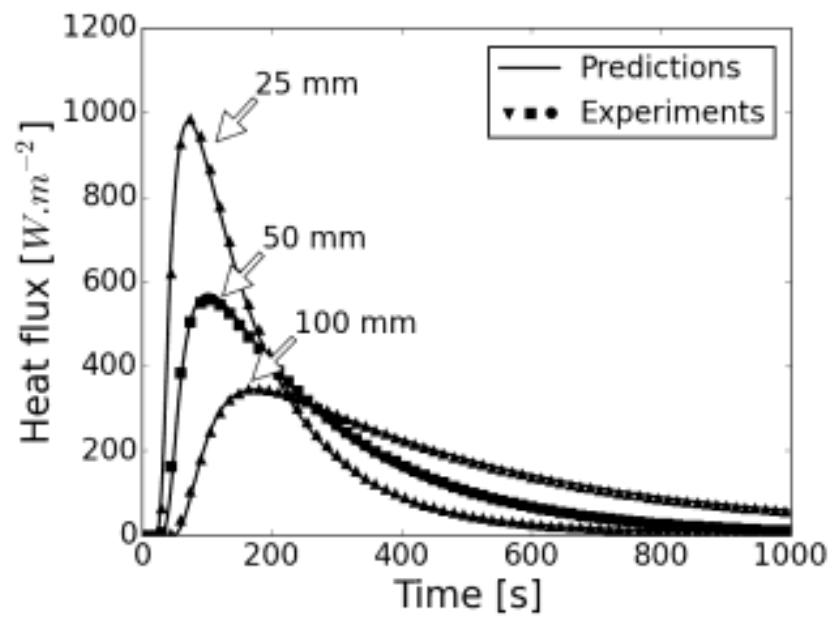

Figure 9: Flux predictions for brass rod tests

This makes it easier to see that the predictions of the heat flux are excellent approximations of the experimentally measured signals. In addition the flux data signals are far less noisy compared to the impulse responses. An important consistency check was added by considering the implications of the energy balance assumption made in the model derivation. If the energy balance holds, the integrals over time of the incoming flux and outgoing flux must be equal. This was used for signal validation to ensure that no drift was present in the data possibly due to ambient effects. As a final validation step the experiments were repeated using aluminium rods. The flux prediction results are given in Figure 10.

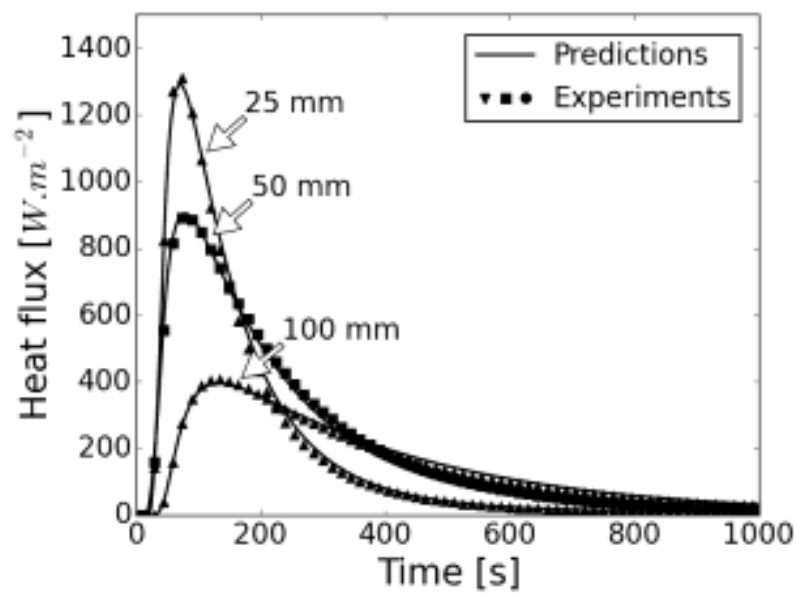

Figure 10: Flux predictions for aluminium rod tests

Again the predictions are very good with an average error of $6.3 \%$ with a standard deviation of $2.7 \%$. The slightly higher error is to be expected given the uncertainty in 
the material thermal conductivity value given by the supplier. Nonetheless the results validate the approach and further verify that the convective heat transfer coefficient remains constant. As part of the investigation it was found that the $25 \mathrm{~mm}$ aluminium rod could be accurately approximated by the theoretical model with only a single term $(n=1)$, as demonstrated in Figure 11.

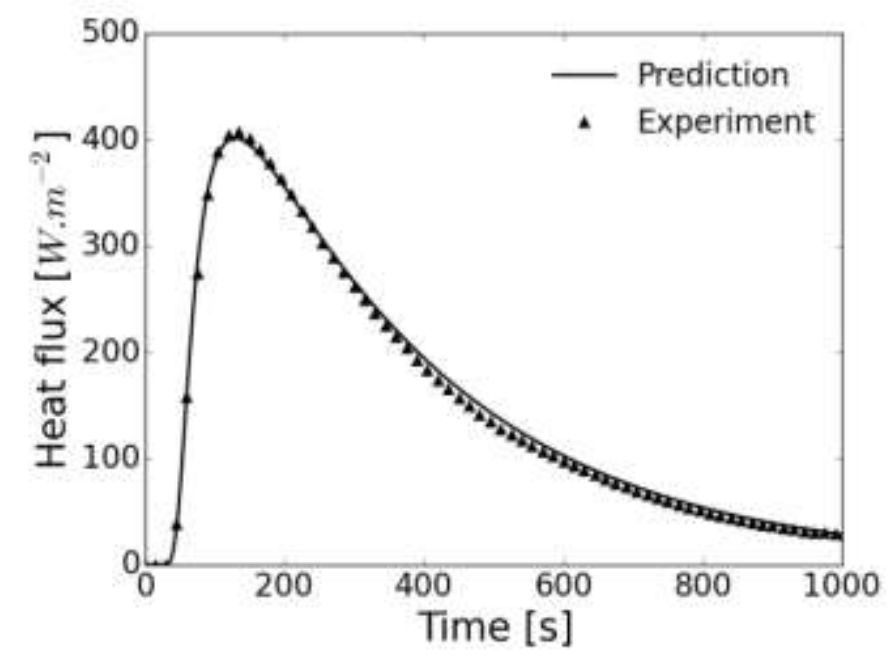

Figure 11: Flux predictions for $25 \mathrm{~mm}$ aluminium rod with $n=1$

For this configuration the Biot number of the system can be calculated as 0.14. Thus this observation is in accordance with the earlier finding that a single term expansion is equivalent to the lumped capacitance approach as a Biot number of $<0.1$ is used as the validity check for applying this approach. Whilst a single term expansion does offer faster convergence of the optimisation step, there is no noticeable difference in the parameter estimation time compared to the six term expansion used as the standard.

The time required for a single experimental measurement is comparatively short. As can be seen from Figure 9 and 10, less than 20 minutes (1200 s) is required to obtain a good parametric fit through the optimisation. This may be further reduced by optimizing the test input signal, i.e. magnitude and duration. Furthermore, since the input signal is directly measured the system does not require a steady state situation at the start of the measurement. Thus there is zero waiting time once the sample has been loaded into the device and given the setup configuration, no sample preparation is needed. This makes the total time for obtaining a parameter estimate every short. 


\section{Conclusions and recommendations}

Rapid thermal property measurement of porous solids and composites remains a challenge. While transient methods offer a rapid alternative they suffer from significant drawbacks. Steady state measurements are robust and can measure the flow through a system but require a long time to reach steady state. In this work a novel modification of a widely used steady state technique has been proposed. The approach uses two heat flux sensors instead of one and is unique since the parameter estimation is done through the deconvolution of these signals.

Signal deconvolution leads to the identification of the system impulse response. A detailed derivation of the theoretically expected behaviour has been done. This provides a basis for fitting the measured impulse response. It is demonstrated that just six terms are required for the theoretical model to achieve full convergence. Interestingly it is shown that the first order expansion of the theoretical transient model is equivalent to the lumped capacitance method. This is confirmed through a small aluminium sample which demonstrates this behaviour and has a Biot number of around 0.1 .

The unit requires a calibration step to measure the convective boundary condition. The results have shown that this value remains constant throughout further testing as expected. A signal validity check has been built into the approach through the use of the energy balance. This ensures that any drift due to ambient losses or other factors is detected. Through suitable choice of the root finding equation the eigenvalues can be readily determined using Ridder's method. The choice of the L-BFGS optimisation algorithm ensures rapid convergence of the non-linear fitting procedure. The parameter estimates of the standard test samples are excellent, with average errors of $2.3 \%$ for brass and $6.3 \%$ for aluminium.

Thus the approach has been validated and is in agreement with the theoretically expected behaviour. The system has several advantages in addition to the short measurement time, including low cost and a very small temperature excursion experienced by the sample. This means that no guard furnace and minimal insulation is required for room temperature measurements. It may be possible to further reduce the measurement time by changing the input power, the only requirement is maintaining a suitable signal to noise ratio. Future work will focus on determining the optimal excitation signal for rapid and accurate property estimation. In addition the 
work will be extended to porous solids and composites. For these materials the method is can be used for the measurement of an overall thermal conductivity for the entire composite with any additional modifications.

\section{Appendix A : Theoretical derivation of model}

A solution to the general heat equation is sought:

$$
\frac{\partial \mathrm{u}}{\partial \mathrm{t}}=\alpha \nabla^{2} \mathrm{u}
$$

Since heat flow into the cylinder is assumed to be invariant, this reduces to a one dimensional problem for finding $\mathrm{u}(\mathrm{x}, \mathrm{t})$ where:

$$
\mathrm{u}_{\mathrm{t}}=\alpha \mathrm{u}_{\mathrm{xx}}
$$

In this case the subscript indicates a derivative with respect to that variable. Once the solution for $u$ is found, the value of $u_{x}$ can be determined, as measured by the flux sensor at $\mathrm{x}=\mathrm{L}$. In order to obtain the response of the system to an arbitrary incoming flux signal, the impulse response of the system can be convolved with any given input signal [20]. The impulse response can be obtained as the time derivative of the unit step response, which is:

$$
-\mathrm{u}_{\mathrm{x}}(0, \mathrm{t})= \begin{cases}0, & \mathrm{t} \leq 0 \\ 1, & \mathrm{t}>0\end{cases}
$$

With the initial condition, $u(x, 0)=0$ and boundary conditions $-u_{x}(0, t)=1$ and $u_{x}(L, t)=-\frac{h}{k} u(L, t)$, the final solution may be stated as [24]:

$$
\mathrm{u}(\mathrm{x}, \mathrm{t})=\mathrm{w}(\mathrm{x}, \mathrm{t})+\mathrm{v}(\mathrm{x})
$$

Where $\mathrm{w}$ is the transient solution and $\mathrm{v}$ is the steady state solution. Assuming $\mathrm{v}(\mathrm{x})=\mathrm{ax}+\mathrm{b}$, during the initial phase one has from the first boundary condition $\mathrm{a}=-1$ and from the second that $\mathrm{b}=\mathrm{kh}^{-1}+\mathrm{L}$, thus:

$$
\mathrm{v}(\mathrm{x})=\mathrm{L}+\frac{\mathrm{k}}{\mathrm{h}}-\mathrm{x}
$$

Using this expression the problem may be transformed into a homogenous mixed boundary problem in $\mathrm{w}$ as follows:

From the initial condition:

$$
u(x, 0)=0=w(x, 0)+v(x)=w(x, 0)+L+\frac{k}{h}-x
$$




$$
\therefore \mathrm{w}(\mathrm{x}, 0)=\mathrm{x}-\mathrm{L}-\frac{\mathrm{k}}{\mathrm{h}}
$$

From the first boundary condition:

$$
\begin{gathered}
-\mathrm{u}_{\mathrm{x}}(0, \mathrm{t})=1=-\mathrm{w}_{\mathrm{x}}(0, \mathrm{t})-\mathrm{v}_{\mathrm{x}}(0, \mathrm{t})=-\mathrm{w}_{\mathrm{x}}(0, \mathrm{t})+1 \\
\therefore \mathrm{w}_{\mathrm{x}}(0, \mathrm{t})=0
\end{gathered}
$$

Finally, from the second boundary condition:

$$
\begin{aligned}
\mathrm{u}_{\mathrm{x}}(\mathrm{L}, \mathrm{t}) & =-\frac{\mathrm{h}}{\mathrm{k}} \mathrm{u}(\mathrm{L}, \mathrm{t}) \\
\mathrm{w}_{\mathrm{x}}(\mathrm{L}, \mathrm{t})+\mathrm{v}_{\mathrm{x}}(\mathrm{L}) & =-\frac{\mathrm{h}}{\mathrm{k}}(\mathrm{w}(\mathrm{L}, \mathrm{t})+\mathrm{v}(\mathrm{L})) \\
\mathrm{w}_{\mathrm{x}}(\mathrm{L}, \mathrm{t})-1=-\frac{\mathrm{h}}{\mathrm{k}}\left(\mathrm{w}(\mathrm{L}, \mathrm{t})+\frac{\mathrm{k}}{\mathrm{h}}\right) & \\
\therefore \mathrm{w}_{\mathrm{x}}(\mathrm{L}, \mathrm{t}) & =\frac{\mathrm{h}}{\mathrm{k}} \mathrm{w}(\mathrm{L}, \mathrm{t})
\end{aligned}
$$

Since the derivative of the steady state solution is zero, the complete problem is given by $\mathrm{w}_{\mathrm{t}}-\alpha \mathrm{w}_{\mathrm{xx}}=0$ with initial condition $\mathrm{w}(\mathrm{x}, 0)=\mathrm{x}-\mathrm{L}$ and boundary conditions $\mathrm{w}_{\mathrm{x}(0, \mathrm{t})}=0$ and $\mathrm{w}_{\mathrm{x}}(\mathrm{L}, \mathrm{t})=-\frac{\mathrm{h}}{\mathrm{k}} \mathrm{w}(\mathrm{L}, \mathrm{t})$.

Now it may be assumed that $\mathrm{w}(\mathrm{x}, \mathrm{t})=\mathrm{X}(\mathrm{x}) \cdot \mathrm{T}(\mathrm{t})$, such that $\mathrm{w}_{\mathrm{t}}=\mathrm{X} . \mathrm{T}^{\prime}$ and $\mathrm{w}_{\mathrm{X}}=\mathrm{X}^{\prime \prime} \cdot \mathrm{T}$. The original problem becomes $\mathrm{X} \cdot \mathrm{T}^{\prime}=\alpha \mathrm{X}^{\prime \prime} \cdot \mathrm{T}^{\prime}$. Through the separation of variables one finds:

$$
\frac{\mathrm{X}^{\prime \prime}}{\mathrm{X}}=\frac{1}{\alpha} \frac{\mathrm{T}^{\prime}}{\mathrm{T}}=-\lambda^{2}
$$

It may be easily demonstrated that a zero value or different sign leads only to trivial solutions. This leads to two separate ordinary differential equations:

$$
\mathrm{X}^{\prime \prime}+\lambda^{2} \mathrm{X}=0 \text { and } \mathrm{T}+\lambda^{2} \alpha \mathrm{T}^{\prime}=0
$$

With the general solutions:

$$
X=c_{1} \cos (\lambda x)+c_{2} \sin (\lambda x) \text { and } Y=c_{3} e^{-\lambda^{2} \alpha t}
$$

Therefore,

$$
\begin{aligned}
\mathrm{w} & =\mathrm{XT} \\
& =\mathrm{c}_{3} \mathrm{e}^{-\lambda^{2} \alpha \mathrm{t}}\left[\mathrm{c}_{1} \cos (\lambda \mathrm{x})+\mathrm{c}_{2} \sin (\lambda \mathrm{x})\right] \\
& =\mathrm{e}^{-\lambda^{2} \alpha \mathrm{t}}[\mathrm{A} \cos (\lambda \mathrm{x})+\mathrm{B} \sin (\lambda \mathrm{x})]
\end{aligned}
$$


Next the boundary conditions may be applied to solve for the unknown constants. From the first boundary condition $\mathrm{w}_{\mathrm{x}}(0, \mathrm{t})=0 \rightarrow-\mathrm{e}^{-\lambda^{2} \alpha \mathrm{t}}[\mathrm{A} \lambda \sin (\lambda 0)+$ $\mathrm{B} \lambda \cos (\lambda 0)]=0 \rightarrow \mathrm{B}=0$, so $\mathrm{w}=\mathrm{Ae}^{-\lambda^{2} \alpha \mathrm{t}} \cos (\lambda \mathrm{x})$.

Now, from the second boundary condition, $w_{x}(L, t)=-\frac{h}{k} w(L, t)$,

$$
\begin{aligned}
-\mathrm{Ae}^{-\lambda^{2} \alpha \mathrm{t}} \lambda \sin (\lambda \mathrm{L}) & =-\frac{\mathrm{h}}{\mathrm{k}} \mathrm{Ae}^{-\lambda^{2} \alpha \mathrm{t}} \cos (\lambda \mathrm{L}) \\
\lambda \sin (\lambda \mathrm{L}) & =\frac{\mathrm{h}}{\mathrm{k}} \cos (\lambda \mathrm{L})
\end{aligned}
$$

Assuming a linear combination of these specific solutions can satisfy the initial condition, the constants $A_{n}$ may then be determined as follows:

$$
w(x, 0)=x-\left(L+\frac{k}{h}\right)=\sum_{n=1}^{\infty} A_{n} \cos \left(\lambda_{n} x\right)
$$

Multiplying both sides by $\cos \left(\lambda_{\mathrm{n}} \cdot \mathrm{x}\right)$ and integrating along the length of the rod (0 to L) gives:

$$
\int_{0}^{L} \cos \left(\lambda_{m} x\right) x-\left(L+\frac{k}{h}\right) \cos \left(\lambda_{m} x\right) d x=A_{n} \sum_{n=1}^{\infty} \int_{0}^{L} \cos \left(\lambda_{n} x\right) \cos \left(\lambda_{m} x\right) d x
$$

The integral on the right vanishes except when $\mathrm{n}=\mathrm{m}$, thus one has:

$$
\int_{0}^{L} \cos \left(\lambda_{n} x\right) x d x-\left(L+\frac{k}{h}\right) \int_{0}^{L} \cos \left(\lambda_{n} x\right) d x=A_{n} \sum_{n=1}^{\infty} \int_{0}^{L} \cos ^{2}\left(\lambda_{n} x\right) d x
$$

Which evaluates to:

$$
\frac{\mathrm{L} \lambda_{\mathrm{n}} \sin \left(\mathrm{L} \lambda_{\mathrm{n}}\right)+\cos \left(\mathrm{L} \lambda_{\mathrm{n}}\right)-1}{\lambda_{\mathrm{n}}^{2}}-\frac{\left(\mathrm{L}+\frac{\mathrm{k}}{\mathrm{h}}\right) \frac{1}{\mathrm{~L}} \sin \left(\mathrm{L} \lambda_{\mathrm{n}}\right)}{\lambda_{\mathrm{n}}}=\mathrm{A}_{\mathrm{n}} \frac{2 \mathrm{~L} \lambda_{\mathrm{n}}+\sin \left(2 \mathrm{~L} \lambda_{\mathrm{n}}\right)}{4 \lambda_{\mathrm{n}}}
$$

Solving for $A_{n}$ yields:

$$
A_{n}=-\frac{4\left(k \lambda_{n} \sin \left(L \lambda_{n}\right)-h \cos \left(L \lambda_{n}\right)+h\right)}{2 L h \lambda_{n}^{2}+h \lambda_{n} \sin \left(2 L \lambda_{n}\right)}
$$

But the second boundary condition requires that:

$$
\mathrm{k} \lambda_{\mathrm{n}} \sin \left(\mathrm{L} \lambda_{\mathrm{n}}\right)-\mathrm{h} \cos \left(\mathrm{L} \lambda_{\mathrm{n}}\right)=0
$$

Thus:

$$
A_{n}=-\frac{4}{2 L \lambda_{n}^{2}+\lambda_{n} \sin \left(2 L \lambda_{n}\right)}
$$


In most cases this series was found to converge very rapidly and less than ten terms are required for an error of less than $10^{-6}$. Combining the solution of $\mathrm{w}$ with the solution of $\mathrm{v}$ (equation (5)) gives the final solution of $\mathrm{u}$ :

$$
u(x, t)=L-x+\frac{k}{h}+\sum_{n=1}^{\infty} A_{n} e^{-\lambda_{n}^{2} \alpha t} \cos \left(\lambda_{n} x\right)
$$

This expression can be differentiated with respect to $\mathrm{x}$, to obtain the expression for the heat flux:

$$
u_{x}(x, t)=-1-\sum_{n=1}^{\infty} A_{n} \lambda_{n} e^{-\lambda_{n}^{2} \alpha t} \sin \left(\lambda_{n} x\right)
$$

To obtain the impulse response the derivative with respect to time is taken which gives:

$$
u_{x t}=\sum_{n=1}^{\infty} A_{n} \alpha \lambda_{n}^{3} e^{-\alpha \lambda_{n}^{2} t} \sin \left(\lambda_{n} x\right)
$$

To obtain the flux out of the cylinder $\mathrm{x}$ is simply set to a value of $\mathrm{L}$.

$$
\begin{aligned}
& \text { Nomenclature: } \\
& \mathrm{u} \text { - Temperature } \\
& \mathrm{y} \text { - Position } \\
& \alpha \text { - Thermal diffusivity } \\
& \mathrm{t} \text { - Time } \\
& \mathrm{h} \text { - Convective transfer coefficient } \\
& \mathrm{k} \text { - Thermal conductivity } \\
& \text { L - Total path length } \\
& \lambda \text { - Eigenvalue }
\end{aligned}
$$

\section{References}

[1] J. Klett, R. Hardy, E. Romine, C. Walls, T.S. Burchell, High-thermal-conductivity, mesophase-pitch-derived carbon foams: effect of precursor on structure and properties, Carbon, 38 (2000) 953-973.

[2] Focke WW, Badenhorst H, Ramjee S, Kruger HJ, van Schalkwyk R, Rand B. Graphite foam from pitch and expandable graphite. Carbon, 2014;73:41-50. 
[3] A. Franco, An apparatus for the routine measurement of thermal conductivity of materials for building application based on a transient hot-wire method, Applied Thermal Engineering, 27 (2007) 2495-2504.

[4] M.J. Asseal, M. Dix, K. Gialou, L. Vozar, W.A. Wakeham, Application of the Transient Hot-Wire Technique to the Measurement of the Thermal Conductivity of Solids, International Journal of Thermophysics, 23 (2002) 615-633.

[5] J.H. Blackwell, A transient-flow method for determination of thermal constants of insulating materials in bulk, Journal of Applied Physics, 25 (1954) 137-144.

[6] B.E. Belkerk, M.A. Soussou, M. Carette, M.A. Djouadi, Y. Scudeller, Measuring thermal conductivity of thin films and coatings with the ultra-fast transient hotstrip technique, Journal of Physics D: Applied Physics, 45 (2012) 295303.

[7] U. Hammerschmidt, A New Pulse Hot Strip Sensor for Measuring Thermal Conductivity and Thermal Diffusivity of Solids, International Journal of Thermophysics, 24 (2003) 675-682.

[8] S.E. Gustafsson, E. Karawacki, M.N. Khan, Transient hot-strip method for simultaneously measuring thermal conductivity and thermal diffusivity of solids and fluids, Journal of Physics D : Applied Physics, 12 (1979) 1411-1421.

[9] A. Dupleix, A. Kusiak, M. Hughes, F. Rossi, Measuring the thermal properties of green wood by the transient plane source (TPS) technique, Holzforschung, 67 (2012) 437-445.

[10] M. Gustavsson, E. Karawacki, S.E. Gustafsson, Thermal conductivity, thermal diffusivity and specific heat of thin samples from transient measurements with hot disc sensors, Review of Scientific Instruments, 65 (1994) 3856-3859.

[11] S.E. Gustafsson, Transient plane source techniques for thermal conductivity and thermal diffusivity measurements of solid materials, Review of Scientific Instruments, 62 (1991) 797-804.

[12] B.-K. Jang, Y. Sakka, N. Yamaguchi, H. Matsubara, H.-T. Kim, Thermal conductivity of EB-PVD ZrO2-4mol\% Y2O3 films using the laser flash method, Journal of Alloys and Compounds, 509 (2011) 1045-1049.

[13] A. Cai, L.-P. Yang, J.-P. Chen, T.-G. Xi, S.-G. Xin, W. Wu, Thermal conductivity of anodic alumina film at (220 to 480) K by laser flash technique, Journal of Chemical and Engineering Data, 55 (2010) 4840-4843. 
[14] D.G. Cahill, Thermal conductivity measurement from 30 to $750 \mathrm{~K}$ : the $3 \omega$ method, Review of Scientific Instruments, 61 (1990) 802-808.

[15] B.-Y. Cao, Y.-W. Li, J. Kong, H. Chen, Y. Xu, K.-L. Yung, A. Cai, High thermal conductivity of polyethylene nanowire arrays fabricated by an improved nanoporous template wetting technique, Polymer, 52 (2011) 1711-1715.

[16] C. Jensen, C. Xing, C. Folsom, H. Ban, J. Phillips, Design and Validation of a High-Temperature Comparative Thermal-Conductivity Measurement System, International Journal of Thermophysics, 33 (2012) 311-329.

[17] R. Wulf, G. Barth, U. Gross, Intercomparison of Insulation Thermal Conductivities Measured by Various Methods, International Journal of Thermophysics, 28 (2007) 1679-1692.

[18] U. Hammerschmidt, W. Sabuga, Transient Hot Strip (THS) Method: Uncertainty Assassment, International Journal of Thermophysics, 21 (2000) 217-248.

[19] M.H. Rausch, K. Krzeminski, A. Leipertz, A.P. Fröba, A new guarded parallelplate instrument for the measurement of the thermal conductivity of fluids and solids, International Journal of Heat and Mass Transfer, 58 (2013) 610-618.

[20] R. Bracewell, The Fourier Transform and Its Applications, McGraw-Hill, New York, 1965

[21] Ridders CFJ, A New Algorithm for Computing a Single Root of a Real Continuous Function. IEEE Trans. Circuits Systems 1979;26:979-80.

[22] Byrd RH, Lu P, Nocedal J, A Limited Memory Algorithm for Bound Constrained Optimization. SIAM Journal on Scientific and Statistical Computing 1995;16:1190-208.

[23] Zhu C, Byrd RH, Nocedal J, L-BFGS-B: Algorithm 778: L-BFGS-B, FORTRAN routines for large scale bound constrained optimization. ACM Transactions on Mathematical Software 1997;23: 550-60.

[24] F.P. Incropera, D.P. DeWitt, T.L. Bergman, A.S. Lavine, Fundamentals of Heat and Mass Transfer, John Wiley \& Sons, New Jersey, 2007. 